\title{
Intranasal ciclesonide for allergic rhinitis
}

\author{
Ben Williams \\ William B Smith \\ Frank E Kette \\ Department of Clinical Immunology \\ and Allergy, Royal Adelaide Hospital, \\ Adelaide South Australia
}

\begin{abstract}
Ciclesonide is a novel corticosteroid which is optimized for topical use. It is a pro-drug which is activated locally in the airway mucosa, lipid-conjugated for local retention, and has very high protein binding in circulation leading to low systemic bioavailability. These characteristics should lead to highly selective activity with reduced local and systemic side effects. It has been established as an inhaled medication for asthma and has also been shown in double-blind trials to be efficacious for the treatment of seasonal and perennial allergic rhinitis. However no data have yet demonstrated superiority over existing nasal topical corticosteroids, either in terms of efficacy or adverse effects, and trials have not yet clearly shown efficacy in rhinitis in children. Therefore the place of ciclesonide in the treatment of allergic rhinitis relative to other existing products remains unclear.
\end{abstract}

Keywords: nasal corticosteroid efficacy, ciclesonide, allergic rhinitis

\section{Introduction}

Allergic rhinitis is characterised by chronic inflammation of the nasal membranes with symptoms including itching, sneezing, nasal congestion and rhinorrhoea. An estimated $1 / 5$ of the population have allergic rhinitis, of which $40 \%$ have perennial allergic rhinitis and 40\% have seasonal allergic rhinitis (Meltzer et al 2007). Allergic rhinitis has been shown to decrease quality of life in patients and places a burden on the health care system (Kim et al 2007).

Although accepted treatments for allergic rhinitis include antihistamines, decongestants, anticholinergic agents, intranasal cromolyn and leukotriene receptor antagonists, intranasal corticosteroids are the most effective therapy for allergic rhinitis and firstline therapy in guidelines for moderate to severe disease (Ratner et al 2007). Allergen immunotherapy is also an effective treatment for certain patients with allergic rhinitis. Intranasal corticosteroids include mometasone furoate, beclomethasone diproprionate, fluticasone propionate, fluticasone furoate, triamcinolone acetonide, budesonide and flunisolide (Herman 2007). With the exception of beclomethasone, these agents have been shown to have minimal systemic absorption and side effects and are quickly metabolized to less active metabolites (Herman 2007). In many countries, budesonide, fluticasone, mometasone and triamcinolone are approved for once-daily dosing.

\section{Ciclesonide description}

Ciclesonide is a new-generation corticosteroid that has been developed for treatment of asthma and allergic rhinitis (Ratner et al 2006). Ciclesonide is administered as an inactive pro-drug and converted to active desisobutyryl-ciclesonide (des-CIC) in the upper and lower airways (Ratner et al 2006). In a rat lung model, des-CIC had 100-fold greater affinity for the glucocorticoid receptor (GR) than the parent compound (Stoeck et al 2004). Human GR binding studies showed the same degree of affinity conversion and furthermore demonstrated that the affinity of des-CIC was similar to fluticasone (Belvisi et al 2005). Metabolism of ciclesonide to the active form occurs through the activity of carboxylesterases and cholinesterases and has been demonstrated to 
occur in human nasal epithelial cells in vitro (Sato et al 2007). Des-CIC undergoes reversible lipid conjugation and may serve as a reservoir of active drug, therefore leading to prolonged drug effect (Nave et al 2005). It seems that the conversion rate of ciclesonide to des-CIC is lower in oropharygeal epithelium, which implies a selective activation in respiratory mucosa and therefore an increased corticosteroid effect in the nose (and lung, for the inhaled drug) relative to the oropharynx. This would be expected to lead to reduced steroid-related oropharyngeal and laryngeal side effects (hoarseness, candidiasis) compared to conventional inhaled corticosteroids, and indeed this is seen in clinical studies. Local conversion of ciclesonide to the active metabolite coupled with lipid conjugation and very high protein binding (Rohatagi et al 2005) may serve to limit the systemic corticosteroid effects of the drug; in preclinical studies (in rats) (Belvisi et al 2005), inhaled ciclesonide showed 44-fold reduced potency in inducing adrenal involution and 22-fold in causing femoral plate hypoplasia compared with fluticasone propionate. Oral bioavailability was also shown to be lower relative to budesonide (Stoeck et al 2004).

Formulations of intranasal corticosteroids (INCS) contain preservatives to inhibit bacterial growth, and other additives to retard clearance of the drug and maintain appropriate moisture levels. Marketing of INCS often focuses on differences between products that may be perceived as important in determining prescribing behavior. Both Ciclesonide and budesonide contain potassium sorbate as an inhibitor of bacterial growth. No adverse effects have been reported with the use of potassium sorbate in INCS usage in humans. It does not alter ciliary beat frequency in in vitro studies of cultured human nasal mucosal cells (Hofmann et al 2004). Benzalkonium chloride (BKC) is utilized in all other INCS; data on the effect of BKC on nasal mucosa is controversial. In vitro studies have demonstrated ciliotoxicity with BKC (Riechelmann et al 2004), however reviews of in vivo studies (Graf 2001; Marple et al 2004) have reported no adverse effect on nasal mucociliary clearance or ciliary beat frequency. There has been one report (Naclerio et al 2003) of a decrease in nasal clearance between treatment groups in a 2-week in vivo study of budesonide (no $\mathrm{BKC}$ ) vs mometasone furoate (contains BKC) nasal spray.

Most INCS (except ciclesonide) contain thixotropic agents such as carboxymethylcellulose to confer high viscosity and delay mucosal clearance. These sprays need to be shaken before use to induce shearing forces to decrease viscosity, hence enabling the spray to be administered as a mist. It is not known whether failure to shake the bottle prior to use impairs nasal mucosal distribution and hence effectiveness. In contrast, ciclesonide is presented to the nasal mucosa in a hypotonic suspension (Meltzer 2007). Diffusion of water molecules from the hypotonic solution into the nasal mucosa increases viscosity, delays clearance and hence increases local concentration and absorption of ciclesonide into the nasal mucosa. There is no evidence that the delay in mucosal clearance that occurs with a hypotonic solution offers a clinical advantage over isotonic ICNS formulations containing thixotropic agents. Importantly however, in an in vitro study (Pujara et al 1995) hypotonic solutions induced lactate dehydrogenase release from the rat nasal cavity - a surrogate marker of cell leaching or cell lysis. Ciliary beat frequency does not seem to be impaired in in vitro studies of human nasal mucosa with hypotonic solutions; however similar studies have not been performed in allergic rhinitis, and importantly, not with ciclesonide.

\section{Efficacy}

Ciclesonide has been shown to be effective for treatment of both seasonal and perennial allergic rhinitis. In a placebocontrolled study of 471 patients ciclesonide $200 \mu \mathrm{g}$ has demonstrated a significant reduction in both instantaneous and reflective morning and evening total nasal symptom scores (TNSS) in groups with moderate perennial allergic rhinitis (Meltzer et al 2007). Improvement was made in all four domains of the TNSS - runny nose, itching, sneezing and nasal congestion. Furthermore in a study of 701 patients with moderate seasonal allergic rhinitis, ciclesonide at a dose of $100 \mu \mathrm{g}$ and $200 \mu \mathrm{g}$ has been shown to decrease sum morning and evening reflective TNSS; however the greater dose was associated with a larger improvement in symptom score (Ratner et al 2006). In a placebo-controlled study of 24 patients, ciclesonide has also been shown to increase nasal airflow significantly when measured by anterior rhinomanometry $-30 \%$ increase after 1 week of ciclesonide $200 \mu \mathrm{g}$ daily (Schmidt et al 1999). In a long-term trial of 663 patients to assess the safety of ciclesonide, there was no evidence of tachyphylaxis (Chervinsky et al 2007). There are few studies directly comparing the efficacy of intranasal corticosteroids, and head to head studies designed to assess efficacy have not been published (Herman 2007). Of comparison studies published, most have found similar efficacy between INCS for allergic rhinitis; however there is some evidence that budesonide has superior efficacy to fluticasone in the treatment of seasonal allergic rhinitis (Herman 2007).

\section{Safety and adverse effects}

Ciclesonide has several pharmacokinetic attributes that are desirable from a safety perspective. It has low systemic 
bioavailability ( $<1 \%$ ), rapid clearance (elimination half-life of 3.5 hours) and a highly protein bound active metabolite (99\%) (Nave et al 2006). These features decrease systemic absorption and hence risk for adverse effects. In one pharmacokinetics study (Nave et al 2006), 48 subjects were randomized to 6 groups with a daily dose of 50, 100, 200 and $400 \mu \mathrm{g}$ (od) and $400 \mu \mathrm{g}$ bid ciclesonide. One subject in the $400 \mu \mathrm{g}$ OD and one in the $400 \mu \mathrm{g}$ bid group showed levels of ciclesonide greater than $25 \mathrm{pg} / \mathrm{mL}$ - the lower limit of quantification (LLOQ). For des-CIC, the majority of samples were below the LLOQ $(10 \mathrm{pg} / \mathrm{mL})$; however in the $400 \mu \mathrm{g}$ bd group, 7 out of 324 samples were above $20 \mathrm{pg} / \mathrm{mL}$. Also, there was no decrease in serum free cortisol levels compared with placebo and there was maintenance of diurnal variation in cortisol in those treated with ciclesonide. Although there was a decrease in urinary free cortisol, the decrease occurred in both the ciclesonide and placebo group with no significant difference between these groups. In a study of 471 patients (Meltzer et al 2007), 238 of which were treated with intranasal ciclesonide $200 \mu \mathrm{g}$ daily and 233 with placebo, adverse effects were reported at a similar rate between ciclesonide and placebo group (102 vs 110). Long term data was reported in a 12 month randomised controlled trial of ciclesonide in patients with PAR greater than 12 years of age (Chervinsky et al 2007). 441 patients were assigned to $200 \mu \mathrm{g}$ ciclesonide daily and 222 were assigned to placebo. Reported adverse events were similar in the treatment group and control group $(75.1 \%$ vs $74.3 \%)$ and of similar frequency to those reported for other INCS. Epistaxis, pharyngolaryngeal pain and sinusitis were higher in the treatment group however there was increased nasopharyngitis and upper respiratory tract infection in the placebo group.

All INCS result in an increase in the frequency of epistaxis compared with the use of placebo. Epistaxis is one of the more common adverse effects encountered clinically and may result in cessation of INCS use. Duration of use, method of administration, ascertainment criteria and daily dose are important determinants of this adverse effect. Ciclesonide at a dose of $200 \mu \mathrm{g}$ once daily for 48 weeks resulted in a rate of epistaxis of $10 \%$ compared to $7.2 \%$ in the placebo group (Sato et al 2007). In a 6-week trial, this dose was associated with a rate of epistaxis of $7.6 \%$ compared to a placebo group of 5.2\% (Meltzer et al 2007). The prescribing information for other INCS demonstrates an increase in epistaxis over placebo; however the rates of epistaxis of both groups of subjects for the various INCS are much lower than reported for ciclesonide. It is important to note that the prescribing information for fluticasone propionate
(Flixonase Nasal Drops) at a dose of $400 \mu \mathrm{g}$ identifies a rate of epistaxis of $19 \%$ compared with $4 \%$ in the placebo group (in the treatment of sinonasal polyposis). Intriguingly, at a dose of fluticasone propionate $400 \mu \mathrm{g}$ bd in another study of nasal polyposis, the rate of epistaxis was only $6 \%$, with a rate of $4 \%$ in the placebo group. This illustrates the difficulty in comparing data from one trial to another, even when using the same INCS. Head to head studies for given indications of use are needed to clarify not only efficacy, but also the relative frequency of the more common adverse events. Uncommon adverse events such as septal perforation are likely to be reported only in the post-marketing period. There are no reports of septal perforation with the use of ciclesonide for allergic rhinitis, but any report of epistaxis from a patient using an INCS should alert the clinician to the possibility of significant mucosal ulceration/atrophy. The chronic administration of topical steroids is well recognized to result in atrophy of epithelial surfaces, and concern has been expressed that mucosal atrophy might arise with the long-term use of INCS; evidence for this is lacking, yet concern at the possibility of the development of atrophy has possibly resulted in a degree of overcautiousness in dose and duration of treatment with INCS. Histopathological studies of nasal mucosa before and after long term INCS usage has not been reported for ciclesonide, however nasal mucosal biopsies before and after 12 months of mometasone furoate $200 \mu \mathrm{g} /$ day did not reveal any changes of atrophy or epithelial thickness, but showed a reduction of the inflammatory cell infiltrate and a decrease in focal metaplasia (Minshall et al 1998), and biopsies after 2 to 5 years of continuous treatment with budesonide also did not show any deleterious change (Pipkorn et al 1988). Further studies at higher doses for all of the INCS in a variety of disorders are needed to enable clinicians to confidently use this class of medications to their fullest potential.

Severe adverse events were found to be increased in the ciclesonide group compared with placebo $(13.4 \%$ vs $11.7 \%$ ), but these were not considered to be related to the active medication (Chervinsky et al 2007). Importantly, in terms of systemic adverse events, there was no decrease from baseline or significant difference between the two groups in 24-hour urinary cortisol level or morning plasma cortisol levels at 24 or 48 weeks (Chervinsky et al 2007) of ciclesonide $200 \mu \mathrm{g}$ daily. An absence of adrenal suppression has also been reported for budesonide, triamcinolone and mometasone (Wilson et al 1998). Importantly, the use of betamethasone nasal drops has been reported to affect growth (Daman Willems et al 1994; Skoner et al 2000), although the 
later study did not have age or height controls. No studies have examined growth velocity in children treated with nasal ciclesonide; however an inhaled dose of $160 \mathrm{mg}$ daily (to treat asthma) in children aged 4 to 11 years did not show any modification of adrenal function (Gelfand et al 2006) or any significant change in growth of children aged 5 to 8 over a period of 1 year (Skoner et al 2008). The possible increase in incidence of cataracts and/or glaucoma in association with the use of intranasal corticosteroids remains controversial. Ciclesonide used regularly for 1 year in 318 subjects was not associated with any increase intraocular pressure or cataract development compared with placebo (156 subjects) (Chervinsky et al 2007).

Considering that allergic rhinitis often occurs in patients with asthma, safety when used in combination with standard asthma therapy is an important consideration. In a randomized, double-blind, placebo- and active-controlled noninferiority trial, patients aged 18 to 60 years with asthma and SAR were commenced on a 10 day run-in period of inhaled beclomethasone dipropionate $320 \mu \mathrm{g}$ twice daily and commenced on a 43 day period of either intranasal ciclesonide $200 \mu \mathrm{g}$ or placebo and at the conclusion of this period were given $2 \mathrm{mg}$ of dexamethasone. Fifty-six patients were assigned to ciclesonide and 55 were treated with placebo, and although plasma cortisol $\left(\mathrm{AUC}_{0-24 \mathrm{~h}}\right)$ decreased significantly in the 10-day run-in period of inhaled beclomethasone and intranasal placebo, there was no significant suppression between the placebo and the ciclesonide group at the end of the trial period. A decrease in plasma cortisol after being given dexamethasone the day following the completion of the ciclesonide or placebo period demonstrated that further inhibition of the HPA axis was indeed possible (Ratner et al 2007). In a similarly structured study in which asthma was treated with inhaled fluticasone-salmeterol 500/50 $\mu$ g, ciclesonide did not add to cortisol suppression (Kim et al 2007).

\section{Tolerability}

Perception of taste, anterior and posterior nasal dripping of spray, and smell are important criteria in patient preference for the use of INCS (Mahadevia et al 2004). BKC has an unpleasant bitter taste; budesonide (which does not contain BKC) was preferred over fluticasone propionate on the basis of taste (Shah et al 2003), and similarly, triamcinolone acetonide was preferred over fluticasone propionate and mometasone furoate (Bachert et al 2002). Studies of ciclesonide nasal spray have shown no difference to placebo in parameters of tolerability such as local irritation (Meltzer et al 2007) or discomfort (Chervinsky et al 2007), but taste and smell and other subjective patient preference aspects were not reported.

\section{Discussion}

In a meta-analysis of the efficacy of the treatment of allergic rhinitis, INCS were shown to have a clear benefit over oral antihistamines in relieving nasal symptoms (Weiner et al 1998). Ciclesonide is a novel corticosteroid distinguished by the requirement for conversion from a relatively inactive pro-drug to a potent agent in the mucosa of the target organ, the formation of lipid conjugates leading to prolonged duration of effect, and high protein binding and first-pass metabolism leading to very low systemic absorption when used topically. These features would be expected in theory to increase activity of the drug in the target therapeutic area relative to the areas responsible for adverse effects such as the oropharyngeal mucosa and the systemic circulation. However even if these theoretical advantages were borne out in vivo, it is clear that they are most relevant to the inhalation of corticosteroids to treat asthma, where large doses are delivered to a large area of mucosal epithelium and there is great exposure of the oropharynx during the inhalation procedure. By comparison, when topical corticosteroids are used to treat rhinitis the dose is low and there is minimal exposure of the oropharynx, such that candidiasis is very rare as a side effect even with existing agents.

The distinguishing features of the formulation of ciclesonide nasal spray are the hypotonic solution and the use of potassium sorbate (rather than the more common BKC) as a preservative. It is suggested that the former feature may enhance local concentration and absorption, which should increase efficacy. The lack of BKC may reduce ciliary toxicity (although this is controversial) and improve tolerability.

Although corticosteroids are proven to be more effective than oral antihistamines for the treatment of allergic rhinitis, there is in contrast no convincing evidence that any INCS is more effective than any other (Waddell et al 2003). Several trials have compared some of the available INCS but no published studies have compared ciclesonide with any other INCS. The magnitude of effect of ciclesonide has been reported to be comparable to that previously reported for mometasone (Ratner et al 2006). Clearly there is a need for head to head studies of the various INCS not only to compare efficacy, but also local and systemic adverse effects and nasal tolerability. Based on currently available trials there is no evidence that the unique pharmacological features of ciclesonide increase its efficacy in comparison with other 
available nasal topical corticsteroids, and at this stage it would not seem to offer any clear clinical advantage.

Trials of other INCS have demonstrated efficacy in other related parameters such as ocular symptoms and quality of life; these are not yet available for ciclesonide. Some forms of chronic rhinosinusitis and particularly nasal polyposis are corticosteroid responsive and topical corticosteroids have a demonstrated role in these conditions, but once again this effect has not yet been demonstrated for ciclesonide. Further trials in these areas are awaited. It is possible that the maximum efficacy of topical corticosteroids has already been achieved; in many patients regular use provides major and even total symptom relief. However a significant proportion, particularly those with more complex disease (nasal polyposis, chronic rhinosinusitis), remain symptomatic despite maximal doses of any topical drug. In these cases the limitations to efficacy may have more to do with the system of delivery than the drug. It is known that oral (systemically delivered) corticosteroids can be of major benefit in these conditions (Hissaria et al 2006) but clearly are not safe for long-term treatment. It is possible that different delivery systems such as drops instilled in the head-inverted position may improve efficacy but comparative trials have not been done.

In summary, several novel features distinguish ciclesonide from existing INCS, including characteristics of the drug itself and of its formulation. Although these features may theoretically enhance the safety of the product, there has as yet been no conclusive demonstration that this translates to actual clinically important safety benefit. The theoretical safety advantages of ciclesonide would appear to be more relevant to the treatment of asthma than rhinitis. Although there are as yet no direct comparisons of ciclesonide with other INCS, there does not appear to be indication from the existing studies that it is likely to be more efficacious than other INCS, and there remains doubt about its efficacy in children. Issues such as cost effectiveness, patient choice, licensing and regulation as well as ultimate determination of efficacy and safety relative to existing products may eventually determine the place of ciclesonide in the therapeutic armamentarium and in the marketplace.

\section{Disclosures}

The authors have no conflicts of interest to disclose.

\section{References}

Bachert C, El-Akkad T. 2002. Patient preferences and sensory comparisons of three intranasal corticosteroids for the treatment of allergic rhinitis. Ann Allergy Asthma Immunol, 89(3):292-7.

Belvisi MG, Bundschuh DS, Stoeck M, et al. 2005. Preclinical profile of ciclesonide, a novel corticosteroid for the treatment of asthma. J Pharmacol Exp Ther, 314(2):568-74.
Chervinsky P, Kunjibettu S, Miller DL, et al. 2007. Long-term safety and efficacy of intranasal ciclesonide in adult and adolescent patients with perennial allergic rhinitis. Ann Allergy Asthma Immunol, 99(1):69-76.

Daman Willems CE, Dinwiddie R, Grant DB, et al. 1994. Temporary inhibition of growth and adrenal suppression associated with the use of steroid nose drops. Eur J Pediatr, 153(9):632-4.

Gelfand EW, Georgitis JW, Noonan M, et al. 2006. Once-daily ciclesonide in children: efficacy and safety in asthma. J Pediatr, 148(3):377-83.

Graf P. 2001. Benzalkonium chloride as a preservative in nasal solutions: re-examining the data. Respir Med, 95(9):728-33.

Herman H. 2007. Once-daily administration of intranasal corticosteroids for allergic rhinitis: a comparative review of efficacy, safety, patient preference, and cost. Am J Rhinol, 21(1):70-9.

Hissaria P, Smith W, Wormald PJ, et al. 2006. Short course of systemic corticosteroids in sinonasal polyposis: a double-blind, randomized, placebo-controlled trial with evaluation of outcome measures. J Allergy Clin Immunol, 118(1):128-33.

Hofmann T, Gugatschga M, Koidl B, et al. 2004. Influence of preservatives and topical steroids on ciliary beat frequency in vitro. Arch Otolaryngol Head Neck Surg, 130(4):440-5.

Kim K, Quesada J, Szmaydy-Rikken N, et al. 2007. Intranasal ciclesonide coadministration with inhaled fluticasone propionate-salmeterol does not suppress cortisol in allergic rhinitis patients. J Asthma, 44(7):515-20.

Mahadevia PJ, Shah S, Leibman C, et al. 2004. Patient preferences for sensory attributes of intranasal corticosteroids and willingness to adhere to prescribed therapy for allergic rhinitis: a conjoint analysis. Ann Allergy Asthma Immunol, 93(4):345-50.

Marple B, Roland P, Benninger M. 2004. Safety review of benzalkonium chloride used as a preservative in intranasal solutions: an overview of conflicting data and opinions. Otolaryngol Head Neck Surg, 130(1):131-41.

Meltzer EO. 2007. Formulation considerations of intranasal corticosteroids for the treatment of allergic rhinitis. Ann Allergy Asthma Immunol, 98(1):12-21.

Meltzer EO, Kunjibettu S, Hall N, et al. 2007. Efficacy and safety of ciclesonide, 200 microg once daily, for the treatment of perennial allergic rhinitis. Ann Allergy Asthma Immunol, 98(2):175-81.

Minshall E, Ghaffar O, Cameron L, et al. 1998. Assessment by nasal biopsy of long-term use of mometasone furoate aqueous nasal spray (Nasonex) in the treatment of perennial rhinitis. Otolaryngol Head Neck Surg, 118(5):648-54.

Naclerio RM, Baroody FM, Bidani N, et al. 2003. A comparison of nasal clearance after treatment of perennial allergic rhinitis with budesonide and mometasone. Otolaryngol Head Neck Surg, 128(2):220-7.

Nave R, Meyer W, Fuhst R, et al. 2005. Formation of fatty acid conjugates of ciclesonide active metabolite in the rat lung after 4-week inhalation of ciclesonide. Pulm Pharmacol Ther, 18(6):390-6.

Nave R, Wingertzahn MA, Brookman S, et al. 2006. Safety, tolerability, and exposure of ciclesonide nasal spray in healthy and asymptomatic subjects with seasonal allergic rhinitis. J Clin Pharmacol, 46(4):461-7.

Pipkorn U, Pukander J, Suonpaa J, et al. 1988. Long-term safety of budesonide nasal aerosol: a 5.5-year follow-up study. Clin Allergy, 18(3):253-9.

Pujara CP, Shao Z, Duncan MR, et al. 1995. Effects of formulation variables on nasal epithelial cell integrity: biochemical evaluations. Int $J$ Pharm, 114:197-203.

Ratner PH, Darken P, Wingertzahn M, et al. 2007. Ciclesonide and beclomethasone dipropionate coadministration: effect on cortisol in perennial allergic rhinitis. $J$ Asthma, 44(8):629-33.

Ratner PH, Wingertzahn MA, van Bavel JH, et al. 2006. Effectiveness of ciclesonide nasal spray in the treatment of seasonal allergic rhinitis. Ann Allergy Asthma Immunol, 97(5):657-63.

Riechelmann H, Deutschle T, Stuhlmiller A, et al. 2004. Nasal toxicity of benzalkonium chloride. Am J Rhinol, 18(5):291-9.

Rohatagi S, Luo Y, Shen L, et al. 2005. Protein binding and its potential for eliciting minimal systemic side effects with a novel inhaled corticosteroid, ciclesonide. Am J Ther, 12(3):201-9. 
Sato H, Nave R, Nonaka T, et al. 2007. In vitro metabolism of ciclesonide in human nasal epithelial cells. Biopharm Drug Dispos, 28(1):43-50.

Schmidt BM, Timmer W, Georgens AC, et al. 1999. The new topical steroid ciclesonide is effective in the treatment of allergic rhinitis. $J$ Clin Pharmacol, 39(10):1062-9.

Shah SR, Miller C, Pethick N, et al. 2003. Two multicenter, randomized, single-blind, single-dose, crossover studies of specific sensory attributes of budesonide aqueous nasal spray and fluticasone propionate nasal spray. Clin Ther, 25(8):2198-214.

Skoner DP, Maspero J, Banerji D. 2008. Assessment of the long-term safety of inhaled ciclesonide on growth in children with asthma. Pediatrics, 121(1):e1-14.

Skoner DP, Rachelefsky GS, Meltzer EO, et al. 2000. Detection of growth suppression in children during treatment with intranasal beclomethasone dipropionate. Pediatrics, 105(2):E23.
Stoeck M, Riedel R, Hochhaus G, et al. 2004. In vitro and in vivo anti-inflammatory activity of the new glucocorticoid ciclesonide. $J$ Pharmacol Exp Ther, 309(1):249-58.

Waddell AN, Patel SK, Toma AG, et al. 2003. Intranasal steroid sprays in the treatment of rhinitis: is one better than another? J Laryngol Otol, 117(11):843-5.

Weiner JM, Abramson MJ, Puy RM. 1998. Intranasal corticosteroids versus oral $\mathrm{H} 1$ receptor antagonists in allergic rhinitis: systematic review of randomised controlled trials. Bmj, 317(7173):1624-9.

Wilson AM, Sims EJ, McFarlane LC, et al. 1998. Effects of intranasal corticosteroids on adrenal, bone, and blood markers of systemic activity in allergic rhinitis. J Allergy Clin Immunol, 102(4 Pt 1):598-604. 